\title{
Preface
}

\section{Paraesophageal Hernia \\ Repair: A Still-Evolving \\ Operation in Pursuit of \\ Perfection}

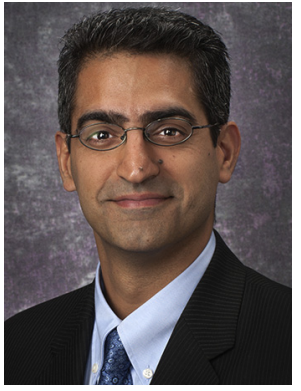

Inderpal S. Sarkaria, MD, FACS

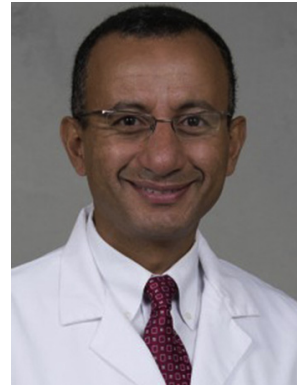

Abbas El-Sayed Abbas, MD, MS, FACS
We are pleased to present this issue of Thoracic Surgery Clinics dedicated to the surgical repair of paraesophageal hernias. Over the decades, our understanding and approach to the management of patients with substantial transhiatal herniation of the stomach and other abdominal viscera into the chest have changed significantly, while simultaneously still adhering to the traditional principles of repair that have stood the test of time. Traditionally approached from the chest by open thoracotomy, abdominal approaches have become the dominant route of repair, with minimally invasive laparoscopic approaches being the route of choice for many surgeons. Robotic approaches have increased in popularity as well, further increasing the operative choices afforded surgeons in caring for this disease.

While many (including the editors) would purport this operative evolution has decreased the morbidity of these operations over time, it would be difficult to argue against the position that proven routes of recurrence and failure still plague our patients to a significant degree. Rates of treatment failures in the form of recurrent herniation or symptomatic reflux continue to be significant sources of morbidity. Also, while often mistakenly thought of as a "simple" hernia repair, practitioners performing these operations come to appreciate the inherent complexity and potential difficulty of these operations in which anatomy can be significantly distorted, and adequate exposure and visualization can be difficult to create and maintain. Furthermore, in these "benign" cases, the simultaneous goal of preserving normal anatomic structures while restoring normal anatomic relationships can prove challenging, as opposed to the resective and exenterative nature of oncologic thoracic surgery. While largely adhering to a set of prime principles, these challenges have otherwise given rise to a rich range of operative approaches, 
technique, and opinion regarding best practices in these cases.

In this issue, we are grateful to have had a truly expert faculty contribute their time and effort to presenting these topics and issues through the selected set of articles. While the articles have been developed to represent relatively unique topics pertinent to these operations, many of the primary principles and tenets of surgery and management are clearly reinforced throughout the readings. The reader will no doubt also find subtle (and sometimes less subtle!) differences in opinion by equally recognized experts in the field. I would urge the reader to approach these articles with the understanding that this speaks to the still-evolving nature of these operations, and the continuing pursuit of surgical perfection to a challenging and complex disease process.

It has been a privilege to work with the authors in assembling this focused issue on paraesophageal hernia repair, and we sincerely hope you enjoy the readings presented.

With warm regards,

Inderpal S. Sarkaria, MD, FACS

Department of Cardiothoracic Surgery University of Pittsburgh Medical Center and University of Pittsburgh School of Medicine

5200 Centre Avenue, Suite 715.27 Pittsburgh, PA 15232, USA

Abbas El-Sayed Abbas, MD, MS, FACS Thoracic Medicine and Surgery Section of Thoracic Surgery Lewis Katz School of Medicine Medicine Education \& Research Building 3500 North Broad Street Philadelphia, PA 19140, USA

E-mail addresses: sarkariais@upmc.edu (I.S. Sarkaria) abbas.abbas@tuhs.temple.edu (A.E.-S. Abbas) 